\title{
Next for EDS? SDD Arrays, GeDDs, and a 40-Year Paradigm Overturned
}

\author{
Richard B. Mott ${ }^{1}$ \\ 1. XrayMaps.Com, Lambertville, NJ.
}

Now that the Silicon Drift Detector (SDD) has become the mainstream technology for SEM/EDS, it's interesting to think about what innovations might emerge. Using multiple SDDs on a single instrument achieves higher rates with lower sum peak artifacts. Research at CSIRO in the 1980s evolved into commercial instruments like the Tescan TIMA and the ThermoFisher (FEI) QEMSCAN and MLA with 2 to 4 SDDs on a column for mineral analysis. The Bruker Quad 5040 put four $10 \mathrm{~mm}^{2}$ SDDs in a conventional tubular package. The "racetrack" or oval configuration improves solid angle by reducing the height of the nose piece, cutting the sample distance [1]. A similar design could use a linear array of smaller devices. A newly published patent application by Schamber et. al. describes multiple X-ray detectors with various geometries and window materials integrated into the chamber of the microscope [2]. Figure 1 shows the Maia detector with 384 devices from Brookhaven National Laboratory. Such density requires custom ASICs for readout electronics and careful attention to preventing crosstalk.

Drift detectors fabricated in high-purity germanium (HPGe) are another interesting idea. Cryogenic planar HPGe X-ray detectors reached resolutions below $110 \mathrm{eV}$ at $\mathrm{Mn}$ but were no better than $\mathrm{Si}(\mathrm{Li})$ at very low energies due to higher capacitance [3][4]. The tiny anode of the SDD geometry solves that problem. An early "GeDD" was built in 1988, just 4 years after the seminal paper on the SDD was published [5]. Its noise FWHM was $120 \mathrm{eV}$, while today's SDDs reach a noise FWHM less than 30eV. A GeDD with similar noise might yield $105 \mathrm{eV}$ at Mn with better quantum efficiency at higher energies. Better cooling would be needed, along with much work on contacts and surface treatment.

Finally, advances in pulse processing can lead to changes in established ideas. It has been received wisdom in quantitative SEM/EDS for 40 years that sum peaks should be restored to the parent peaks, from Statham's 1977 paper to the 4th edition of Goldstein et. al. published this year [6][7]. Yet the two plots in Figure 2 below show otherwise for at least one digital processor. The explanation lies in the treatment of the undetected pile-ups which appear as sum peaks. If the "gap time" or flat top of the usual trapezoidal digital filter is long enough to include both the worst-case rise time from the edge of the SDD and the longest pulse-pair resolving time which is likely to occur, the sum peaks will be Gaussian and appear at their expected energy [8]. In addition, that turns out to be the condition which eliminates the shortfall in dead time due to the cumulative separations between the X-rays in the sum peaks, which are otherwise not included. Sum peak restoration partially corrects for this shortfall, but if the dead time is measured completely and accurately it is not necessary [9].

References:

[1] A. Niculae et. al, Microsc. Microanal. 20 (S3) (2014), p. 1124.

[2] F. Schamber et. al, U.S. Patent Appl. No. 15/224,628, Pub. No. US 2018/0033589 A1, (2018).

[3] P. J. Statham, Microchim. Acta [Suppl.] 15, (1998), p. 1-9.

[4] B. G. Lowe and R. A. Sareen in "Semiconductor X-Ray Detectors", (CRC Press, Boca Raton) p. 266.

[5] P. N. Luke, Nucl. Instr. and Meth. A 271 (1988), p. 567.

[6] P. J. Statham, X-Ray Spectrometry 6(2) (1977), p. 94. 
[7] "Scanning Electron Microscopy and X-Ray Microanalysis", ed. Goldstein et al, 4th ed. (Springer Nature, New York).

[8] R. B. Mott, Nucl. Instr. and Meth. A 624 (2) (2010), p. 265.

[9] The author gratefully acknowledges Owen E. Healy, who analyzed the data in Figure 2 and refused to succumb to proof by appeal to authority. You were right. It just took a while to figure out why.

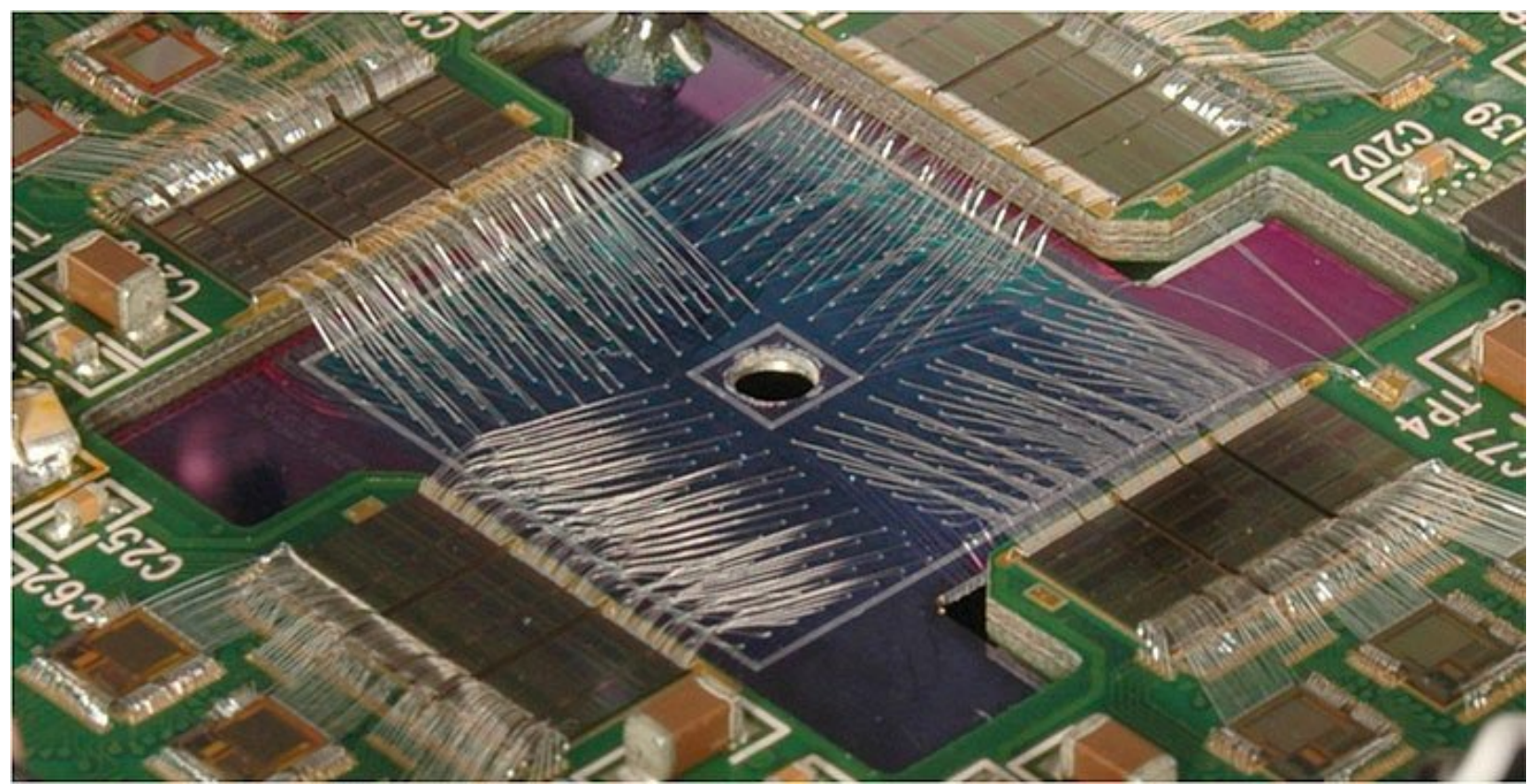

Figure 1. Brookhaven Maia Detector at NSLS-II. 2-inches square, 384 detector elements, each connected to its own readout amplifier. (Image courtesy Brookhaven National Laboratory)
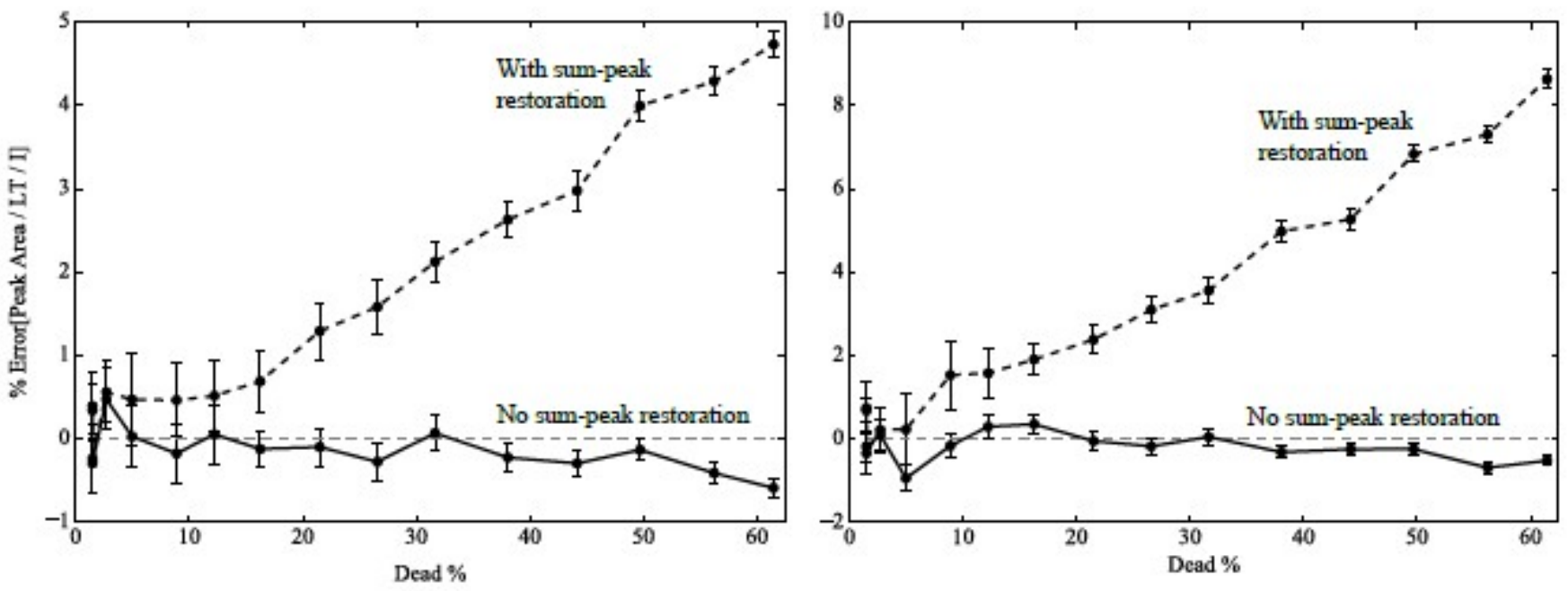

Figure 2. Vertical axis is percent deviation from linearity in peak counts per live second per $\mathrm{nA}$ beam current. The dashed lines are perfect linearity. Horizontal axis is percent dead time. Left-hand plot shows results for $\mathrm{Cu} \mathrm{K}$ line $(8046 \mathrm{eV})$, right-hand plot for $\mathrm{Cu} \mathrm{L}$ line $(930 \mathrm{eV})$. Adding sum peak counts to the parent peak causes increasing errors in linearity at higher dead times. 